\title{
Design of MEMS capacitive accelerometer with different perforated proof- mass for enhancement of performance
}

\author{
Kamran Akhtar, Dr.A. Vimala Juliet \\ Instrumentation and control Engineering SRM University Kattankulathur. Tamilnadu. \\ Instrumentation and control Engineering SRM University Kattankulathur. Tamilnadu
}

\begin{abstract}
This work represents a study of perforated proof mass of a micro fabricated accelerometer with different perforation shapes, by application of load stress and displacement is measuredto know the deflection and flexibility of the proof mass. Perforation shapes of proof mass of capacitive accelerometer affects the sensitivity and other performance parameters of accelerometer.Eigen frequency analysis is done to know the stress and displacement distribution on vibrating proof mass which are used to measure and compare the sensitivity and mechanical strength of proof mass. As a result of this study it has been found that the proof mass with perforation shape of nozzle/diffuser is the most efficient perforation shape to get better performance from an capacitive micro fabricated accelerometers.

Keywords:- Accelerometer, Eigen frequency, MEMS, perforation.
\end{abstract}

\section{Introduction}

Accelerometer is a device used to detect magnitude and direction of the proper acceleration (or g-force), as a vector quantity, and can be used to sense orientation (because direction of weight changes), coordinate acceleration, vibration, shock, and falling in a resistive medium.. Different types of accelerometer are available such as capacitive, piezo-resistive, tunnelling, thermal etc., studies and research on these accelerometer have proved that capacitive accelerometers are more efficient and widely used.Capacitive accelerometer is one of the earliest inertialinstruments studied intensively in MEMS (Micro-Electro-Mechanical Systems) field since 1980s because of its simple structure and easy integration with integrated circuit. Perforation is done to reduce the air damping which has significant effect on the performance Proof mass of capacitive accelerometer will act as movable plates which causes change in distance between the plates so capacitance will change. This change in capacitance will be measured as acceleration.

\section{Device Description}

Proof mass of accelerometer is act as a movable plates when load is applied, proof mass moves causes change in capacitance which can be measured as acceleration.Proof mass with different perforation has different stress and displacement distribution means flexibility and sensitivity of proof mass changes with perforation shapes. $\mathrm{d}$ is distance between the plates when load is applied this distance will change accordingly capacitance will change. Capacitance $\mathrm{C}$ is defined as:-

$$
\mathrm{C}=€ \mathrm{~A} / \mathrm{d}
$$

$€$ iselectrical permittivity of dielectric medium and is defined as $€=€_{0} € \mathrm{r}$,for air as dielectric medium $€=€_{0}$.

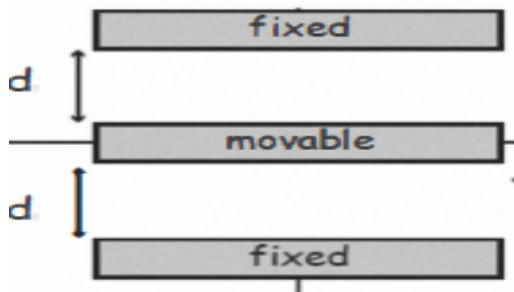

Fig.Equivalent model of capacitive accelerometer

Accelerometer can be modelled as system composed of proof mass,spring,damper.This system can be expressed as:-

$$
\mathrm{F}=\mathrm{ma}=\mathrm{md}^{2} \mathrm{z} / \mathrm{dt}^{2}+\mathrm{bdz} / \mathrm{dt}+\mathrm{kz}
$$

Where $\mathrm{z}$ id displacement, $\mathrm{k}$ is spring constant, $\mathrm{b}$ is damping coefficient and $\mathrm{m}$ is mass of proof mass, a is applied stress. Poly-silicon is used in proof mass as well as in fixed plates since poly -silicon has excellent mechanical and yield strength. 1 kpaloadis applied along negative $\mathrm{z}$-axis. 
Table1:- Model dimension.

\begin{tabular}{|l|l|l|l|}
\hline & $\begin{array}{l}\text { Length in } \\
\mu \mathrm{m}\end{array}$ & $\begin{array}{l}\text { Width in } \\
\mu \mathrm{m}\end{array}$ & $\begin{array}{l}\text { Height in } \\
\mu \mathrm{m}\end{array}$ \\
\hline $\begin{array}{l}\text { Proof } \\
\text { mass }\end{array}$ & 5000 & 5000 & 100 \\
\hline $\begin{array}{l}\text { Square } \\
\text { hole }\end{array}$ & 1000 & 1000 & 100 \\
\hline Anchor & 1500 & 1500 & 100 \\
\hline Beam & 5000 & 1500 & 100 \\
\hline Shin & 3000 & 500 & 100 \\
\hline
\end{tabular}

III. Simulation

All the simulations are done in COMSOL(multi physics), version 4.3and version 4.3a

1) Proof mass with square perforation:-

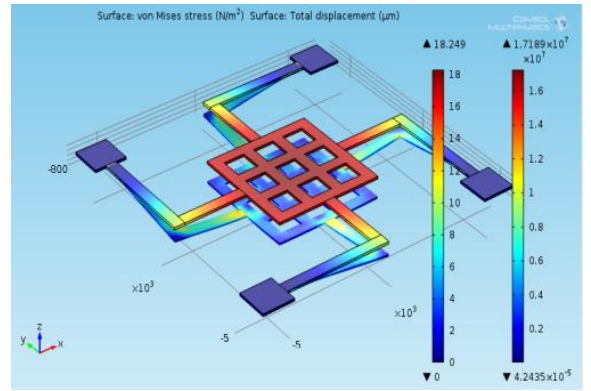

Fig.1:-Proof mass with square perforation

2) Proof mass with cylindricalperforation:-

Radius of circular hole $=500 \mu \mathrm{m}$

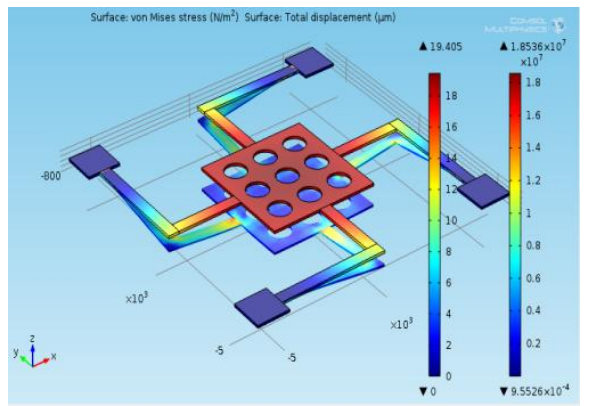

Fig. 2.Proof mass with cylindrical perforation

3) Proof mass with nozzle and diffuser perforation:-

Nozzle a-semi axis $=500 \mu \mathrm{m}$

Nozzle b-semi axis $=500 \mu \mathrm{m}$

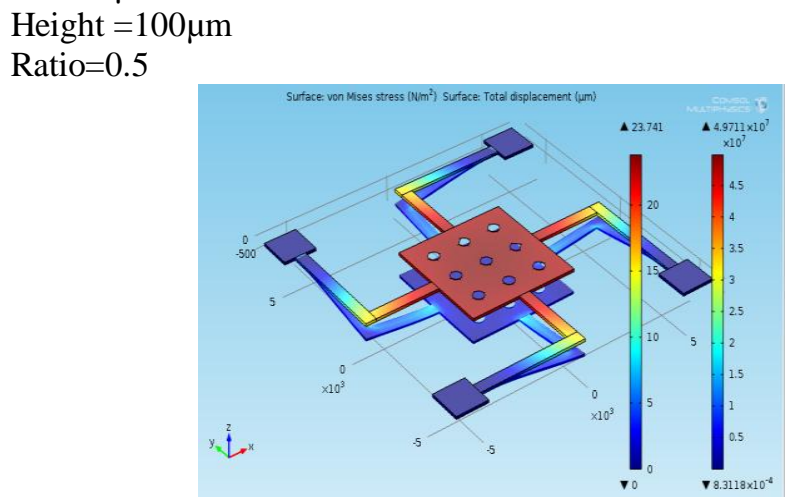

Fig. 3.Proof mass with nozzle diffuser perforation 
4) Proof mass with pyramidal perforation:-

Base length $1=1000 \mu \mathrm{m}$

Base length $2=1000 \mu \mathrm{m}$

Height $=\quad 100 \mu \mathrm{m}$

Ratio $=0.5$

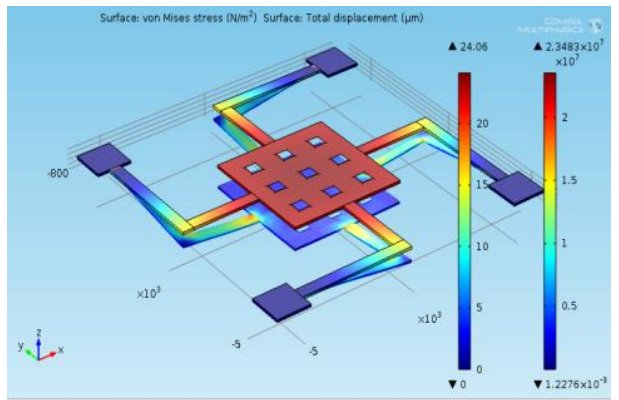

Fig. 4.Proof masswith pyramidal perforation

Table2:- Maximum stress and displacement values On the proof mass of different perforations

\begin{tabular}{|l|l|l|}
\hline Perforation & $\begin{array}{l}\text { Stress in } \\
\mathrm{N} / \mathrm{m}^{\wedge} 2\end{array}$ & $\begin{array}{l}\text { Displacement } \\
\text { in } \mu \mathrm{m}\end{array}$ \\
\hline Square & $1.71 \mathrm{E} 7$ & 18.25 \\
\hline Circle & $1.85 \mathrm{E} 7$ & 19.40 \\
\hline $\begin{array}{l}\text { Nozzle } \\
\text { diffuser }\end{array}$ & $4.97 \mathrm{E} 7$ & 23.74 \\
\hline Pyramidal & $2.34 \mathrm{E} 7$ & 24.06 \\
\hline
\end{tabular}

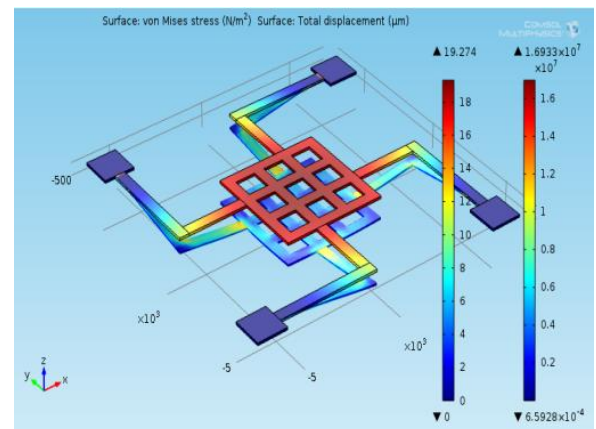

Fig.5:-Proof mass with square perforation and changed beam position change Stress and displacement values will be change if We change the spring position of the proof mass

Table3:- Stress and displacement values with change spring position

\begin{tabular}{|l|l|l|}
\hline Perforation & $\begin{array}{l}\text { Stress in } \\
\mathrm{N} / \mathrm{m}^{\wedge} 2\end{array}$ & $\begin{array}{l}\text { Displacement } \\
\text { in } \mu \mathrm{m}\end{array}$ \\
\hline Square & $1.69 \mathrm{E} 7$ & 19.27 \\
\hline Circle & $2.27 \mathrm{E} 7$ & 21.36 \\
\hline $\begin{array}{l}\text { Nozzle } \\
\text { diffuser }\end{array}$ & $2.63 \mathrm{E} 7$ & 26.32 \\
\hline Pyramidal & $2.35 \mathrm{E} 7$ & 25.03 \\
\hline
\end{tabular}

\section{Eigen Frequency Analysis}

In this analysis proof mass vibrates in six different modes at six different frequencies. These frequencies decides the maximum stress and maximum displacement of the proof mass. These frequencies depend on the model design.

1. Proof mass with cylindrical perforation 
Table4:- Stress and displacement values at six modes.

\begin{tabular}{|l|l|l|l|}
\hline Modes & $\begin{array}{l}\text { Frequency } \\
\text { In Hz }\end{array}$ & $\begin{array}{l}\text { Stress } \\
\text { in N/m² }\end{array}$ & $\begin{array}{l}\text { Displacement } \\
\text { in micro meter }\end{array}$ \\
\hline Mode1 & 2111.25 & $2.28 \mathrm{E} 12$ & $2.35 \mathrm{E} 6$ \\
\hline Mode2 & 4436.62 & $5.46 \mathrm{E} 12$ & $3.51 \mathrm{E} 6$ \\
\hline Mode3 & 4438.59 & $5.54 \mathrm{E} 12$ & $3.53 \mathrm{E} 6$ \\
\hline Mode4 & 8231.76 & $8.50 \mathrm{E} 12$ & $4.43 \mathrm{E} 6$ \\
\hline Mode5 & 9971.85 & $1.11 \mathrm{E} 13$ & $4.58 \mathrm{E} 6$ \\
\hline Mode6 & 13094.56 & $1.88 \mathrm{E} 13$ & $4.17 \mathrm{E} 6$ \\
\hline
\end{tabular}

2. Proof mass with square perforation:-

Table 5:- Stress and displacement values at six modes.

\begin{tabular}{|l|l|l|l|}
\hline Modes & $\begin{array}{l}\text { Frequency } \\
\text { In Hz }\end{array}$ & $\begin{array}{l}\text { Stress } \\
\text { in N/m^2 }\end{array}$ & $\begin{array}{l}\text { Displacement } \\
\text { in micro meter }\end{array}$ \\
\hline Mode1 & 4385.68 & $5.98 \mathrm{E} 12$ & $4.37 \mathrm{E} 6$ \\
\hline Mode2 & 4385.95 & $5.94 \mathrm{E} 12$ & $4.36 \mathrm{E} 6$ \\
\hline Mode3 & 8339.22 & $8.34 \mathrm{E} 12$ & $4.46 \mathrm{E} 6$ \\
\hline Mode4 & 10062.45 & $10.4 \mathrm{E} 12$ & $4.64 \mathrm{E} 6$ \\
\hline Mode5 & 12997.44 & $2.35 \mathrm{E} 13$ & $5.65 \mathrm{E} 6$ \\
\hline Mode6 & 13010.51 & $2.40 \mathrm{E} 13$ & $5.57 \mathrm{E} 6$ \\
\hline
\end{tabular}

3. Proof mass with pyramidal perforation

Table 6:- Stress and displacement values at six modes.

\begin{tabular}{|l|l|l|l|}
\hline Modes & $\begin{array}{l}\text { Frequency } \\
\text { inHz }\end{array}$ & $\begin{array}{l}\text { Stress in } \\
\mathrm{N} / \mathrm{m}^{\wedge} 2\end{array}$ & $\begin{array}{l}\text { Displacement } \\
\text { In micro meter }\end{array}$ \\
\hline Mode1 & 2173.43 & $2.28 \mathrm{E} 12$ & $2.31 \mathrm{E} 6$ \\
\hline Mode2 & 4477.34 & $5.30 \mathrm{E} 12$ & $3.56 \mathrm{E} 6$ \\
\hline Mode3 & 4478.34 & $5.40 \mathrm{E} 12$ & $3.56 \mathrm{E} 6$ \\
\hline Mode4 & 7887.52 & $8.23 \mathrm{E} 12$ & $4.58 \mathrm{E} 6$ \\
\hline Mode5 & 9721.36 & $9.572 \mathrm{E} 12$ & $4.66 \mathrm{E} 6$ \\
\hline Mode6 & 13010.51 & $2.40 \mathrm{E} 13$ & $5.57 \mathrm{E} 6$ \\
\hline
\end{tabular}

4. Proof mass with nozzle diffuser perforation

Table7:- Stress and displacement values at six modes

\begin{tabular}{|l|l|l|l|}
\hline Modes & $\begin{array}{l}\text { Frequency } \\
\text { In Hz }\end{array}$ & $\begin{array}{l}\text { Stress } \\
\text { in N/m^2 }\end{array}$ & $\begin{array}{l}\text { Displacement } \\
\text { in micro meter }\end{array}$ \\
\hline Mode1 & 2028.33 & $3.29 \mathrm{E} 12$ & $2.31 \mathrm{E} 6$ \\
\hline Mode2 & 4376.26 & $5.80 \mathrm{E} 12$ & $3.88 \mathrm{E} 6$ \\
\hline Mode3 & 4379.78 & $5.88 \mathrm{E} 12$ & $3.79 \mathrm{E} 6$ \\
\hline Mode4 & 8875.34 & $27.52 \mathrm{E} 12$ & $4.63 \mathrm{E} 6$ \\
\hline Mode5 & 10375.38 & $16.62 \mathrm{E} 12$ & $4.76 \mathrm{E} 6$ \\
\hline Mode6 & 13199.45 & $28.87 \mathrm{E} 12$ & $5.73 \mathrm{E} 6$ \\
\hline
\end{tabular}

\section{Eigen Frequency Analysis With Spring Position Change}

1. Proof mass with cylindrical perforation:-

Table8:- Stress and displacement distribution.

\begin{tabular}{|l|l|l|l|}
\hline Modes & $\begin{array}{l}\text { Frequency } \\
\text { inHz }\end{array}$ & $\begin{array}{l}\text { Stress in } \\
\mathrm{N} / \mathrm{m}^{\wedge} 2\end{array}$ & $\begin{array}{l}\text { Displacement } \\
\text { In micro meter }\end{array}$ \\
\hline Mode1 & 1938.65 & $6.01 \mathrm{E} 12$ & $3.38 \mathrm{E} 6$ \\
\hline Mode2 & 4401.89 & $6.29 \mathrm{E} 12$ & $4.66 \mathrm{E} 6$ \\
\hline Mode3 & 4624.24 & $6.53 \mathrm{E} 12$ & $4.67 \mathrm{E} 6$ \\
\hline Mode4 & 8305.56 & $9.31 \mathrm{E} 12$ & $5.57 \mathrm{E} 6$ \\
\hline Mode5 & 9871.19 & $12.5 \mathrm{E} 12$ & $5.69 \mathrm{E} 6$ \\
\hline Mode6 & 12415.23 & $19.67 \mathrm{E} 12$ & $5.74 \mathrm{E} 6$ \\
\hline
\end{tabular}


2. Proof mass with nozzle diffuser perforation

Table9:- Stress and displacement distribution.

\begin{tabular}{|l|l|l|l|}
\hline Modes & $\begin{array}{l}\text { Frequency } \\
\text { inHz }\end{array}$ & $\begin{array}{l}\text { Stress in } \\
\mathrm{N} / \mathrm{m}^{\wedge} 2\end{array}$ & $\begin{array}{l}\text { Displacement } \\
\text { In micro meter }\end{array}$ \\
\hline Mode1 & 1940.33 & $2.5 \mathrm{E} 12$ & $2.25 \mathrm{E} 6$ \\
\hline Mode2 & 4453.56 & $7.2 \mathrm{E} 12$ & $4.24 \mathrm{E} 6$ \\
\hline Mode3 & 4453.99 & $7.72 \mathrm{E} 12$ & $4.25 \mathrm{E} 6$ \\
\hline Mode4 & 8246.52 & $1.31 \mathrm{E} 13$ & $5.5 \mathrm{E} 6$ \\
\hline Mode5 & 9931.05 & $1.54 \mathrm{E} 13$ & $5.5 \mathrm{E} 6$ \\
\hline Mode6 & 12630.53 & $3.01 \mathrm{E} 13$ & $6.35 \mathrm{E} 6$ \\
\hline
\end{tabular}

3. Proof mass with square perforation:-

Table 10:- Stress and displacement values at six modes.

\begin{tabular}{|l|l|l|l|}
\hline Modes & $\begin{array}{l}\text { Frequency } \\
\text { inHz }\end{array}$ & $\begin{array}{l}\text { Stress in } \\
\mathrm{N} / \mathrm{m}^{\wedge} 2\end{array}$ & $\begin{array}{l}\text { Displacement } \\
\text { In micro } \\
\text { meter }\end{array}$ \\
\hline Mode1 & 2121.60 & $2.30 \mathrm{E} 12$ & $2.42 \mathrm{E} 6$ \\
\hline Mode2 & 4645.65 & $6.47 \mathrm{E} 12$ & $4.30 \mathrm{E} 6$ \\
\hline Mode3 & 4648.61 & $6.54 \mathrm{E} 12$ & $4.33 \mathrm{E} 6$ \\
\hline Mode4 & 7851.48 & $8.35 \mathrm{E} 12$ & $4.88 \mathrm{E} 6$ \\
\hline Mode5 & 9817.13 & $10.34 \mathrm{E} 12$ & $4.88 \mathrm{E} 6$ \\
\hline Mode6 & 12979.01 & $23.38 \mathrm{E} 12$ & $5.77 \mathrm{E} 6$ \\
\hline
\end{tabular}

4. Proof mass with pyramidal perforation:-

Table11:- Stress and displacement distribution.

\begin{tabular}{|l|l|l|l|}
\hline Modes & $\begin{array}{l}\text { Frequency } \\
\text { inHz }\end{array}$ & $\begin{array}{l}\text { Stress } \\
\text { in } \\
\mathrm{N} / \mathrm{m}^{\wedge} 2\end{array}$ & $\begin{array}{l}\text { Displacement } \\
\text { In micro } \\
\text { meter }\end{array}$ \\
\hline Mode1 & 2019.17 & $2.56 \mathrm{E} 12$ & $2.57 \mathrm{E} 6$ \\
\hline Mode2 & 4560.45 & $6.46 \mathrm{E} 12$ & $4.33 \mathrm{E} 6$ \\
\hline Mode3 & 4562.98 & $6.50 \mathrm{E} 12$ & $4.34 \mathrm{E} 6$ \\
\hline Mode4 & 8346.18 & $8.86 \mathrm{E} 12$ & $4.57 \mathrm{E} 6$ \\
\hline Mode5 & 10196.20 & $1.23 \mathrm{E} 13$ & $4.76 \mathrm{E} 6$ \\
\hline Mode6 & 13056.83 & $2.4 \mathrm{E} 13$ & $5.40 \mathrm{E} 6$ \\
\hline
\end{tabular}

\section{Graphical Representation}

Stress distribution

1. Proof mass with circular perforation:-

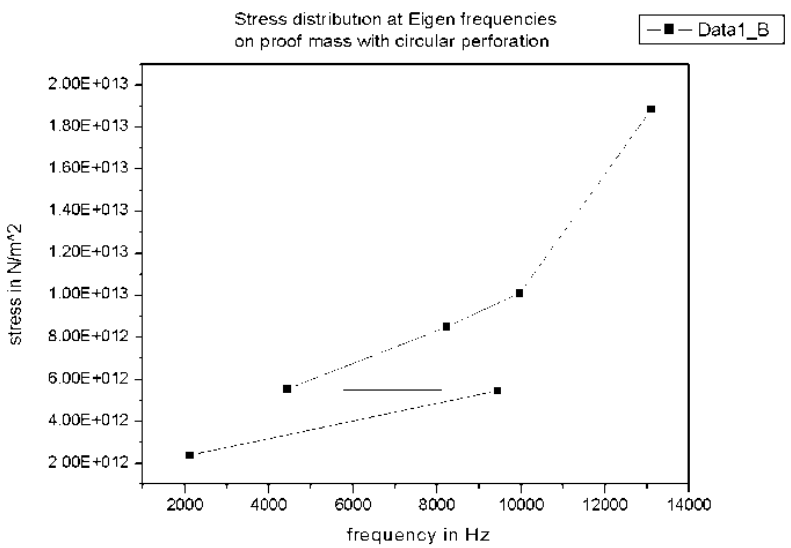


2. Proof mass with square perforation

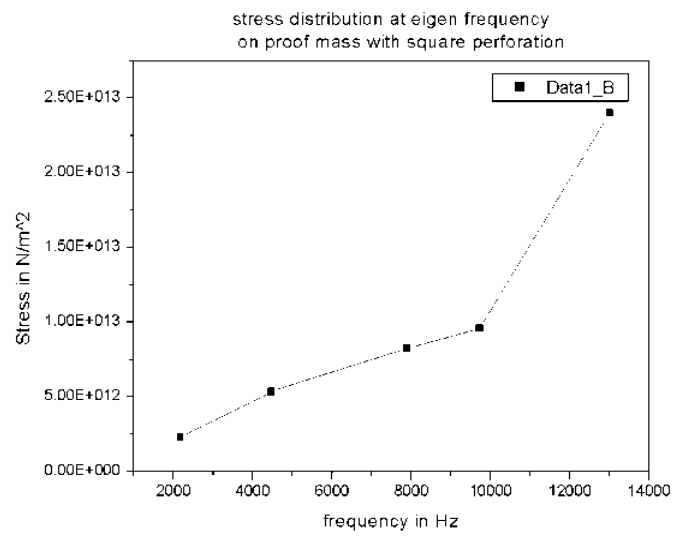

3. Proof mass with nozzle diffuser perforation

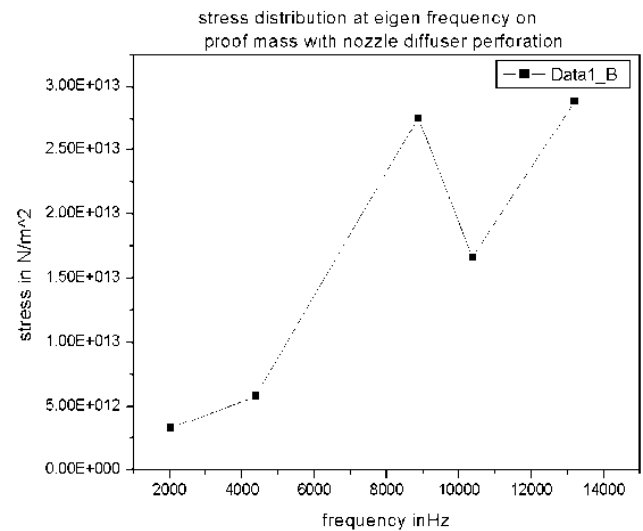

4. Proof mass with pyramidal perforation

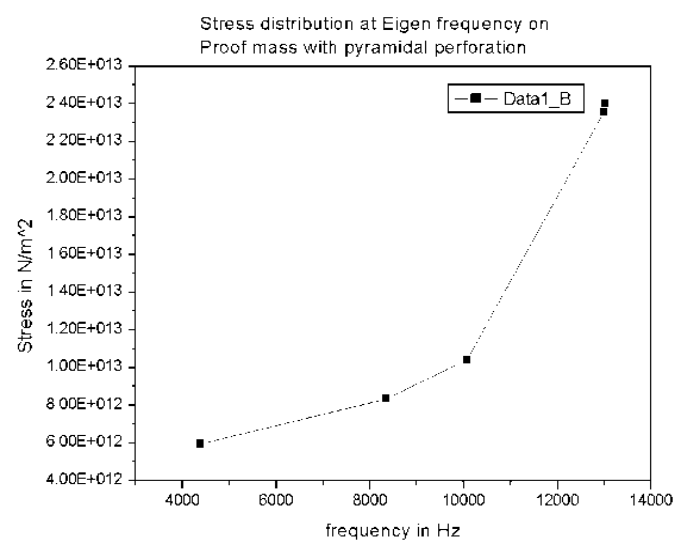

Displacement distribution

1. Proof mass with circular perforation 


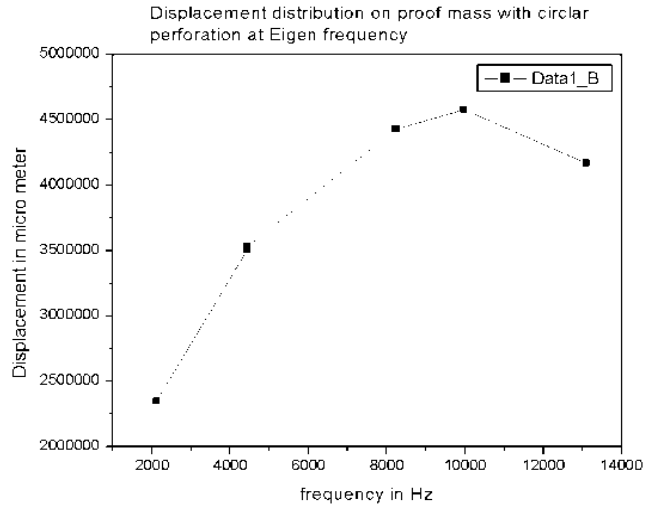

2. Proof mass with square perforation

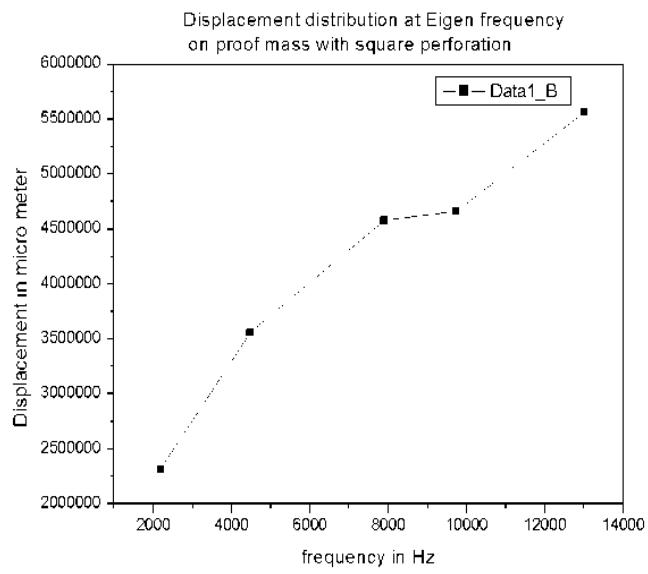

3. Proof mass with nozzle diffuser perforation

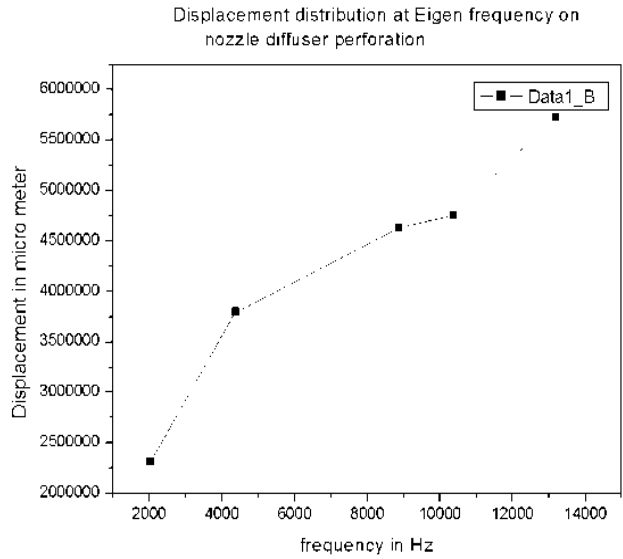

4. Proof mass with pyramidal perforation

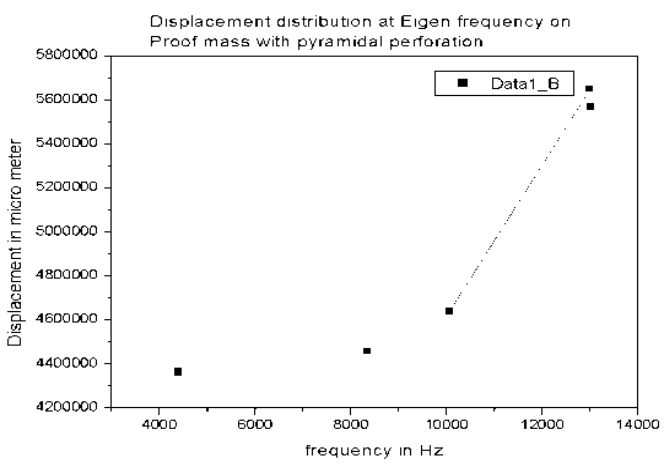




\section{Result}

In the same perforated proof mass if position of spring changes maximum displacement and maximum stress will also change.Maximum stress is observed in proof mass with nozzle-diffuse perforation.Minimum stress is observed in proof mass with square perforation when spring position changes.Maximum displacement is observed in proof mass withnozzle-diffuserperforation when spring position changes.Minimum displacement isobserved in proof mass with square perforation.

\section{Conclusion.}

Maximum Stress and maximum displacement in nozzle diffuser perforation shows that it is more sensitive to stress and displacement on application of load. Minimum stress and displacement in square perforation shows that it is less sensitive to stress and displacement on application of load. So nozzle -diffuser perforated proof mass is more sensitive as well as has better mechanical strength.

\section{References}

[1] Inn am etal"School of Mechanical and Aerospace Engineering, Seoul National University, San 56-1, Shilim-Dong, Kwanak-Gu, Seoul, South Korea "School of Electrical Engineering and Computer Science", Seoul National University, Seoul, South Korea.2004

[2] Isaac G.Macwanet al "Department of Electrical and Computer Engineering, University of Bridgeport", Bridgeport, CT 06604. 2005

[3] BanibrataMukherjeeet al, "Department of Electrical Engineering Institute of Technology, Kharagpur”,Kharagpur-721302.

[4] Jiri et al "Sensor for automotve Technology" Wiley-VCH GmbH \&Co, 2003

[5] G.K.Fedder,"SimulationofMicroelectromechanicalsystem"Ph.D thesis University of California Berkeley.

[6] E.Peeters,S. Vergote,B.Puers and W.Sansen,"A highly symmetrical capacitive micro-accelerometer with singledegree-of-freedom response"J.Miceomech. Microeng

[1] E. Peeters, S. Vergote, B. Puers and W. Sansen," A highly symmetrical

capacitive micro-accelerometer with single degree-of-freedom response"

J.Micromech. Microeng.2(1992) 104-112 